\title{
Efecto de diferentes mulches orgánicos sobre el cultivo de brócoli (Brassica oleracea L. var. Italica) en Ecuador
}

\author{
Effect of different organic mulches on growing broccoli \\ (Brassica oleracea L. var. Italica) in Ecuador \\ Vanessa Frutos ${ }^{1}$, Marco Pérez ${ }^{1}$ y David Risco ${ }^{1} 2^{*}$
}

\section{RESUMEN}

En un cultivo de brócoli (Brassica oleracea L. var. Italica) de los Andes ecuatorianos (2.850 m.s.n.m., $1^{\circ} 24^{\prime}$ S, $\left.78^{\circ} 35^{\prime} \mathrm{O}\right)$, se evaluaron algunas variables de crecimiento, producción en cosecha, control de malezas y contenido de humedad y nitrógeno, como respuesta a la aplicación de diferentes tipos de mulch orgánico. Se utilizó un diseño experimental de bloques al azar con cinco tratamientos y tres repeticiones. Los tratamientos fueron: M0: sin mulch; MM: mulch de maíz (Zea mays L.); MV: mulch de vicia (Vicia sativa L.); MA: mulch de avena (Avena sativa L.) y MC: mulch de caña de azúcar (Saccharum officinarum L.). El crecimiento vegetativo y la producción en cosecha fueron superiores $(\mathrm{p}<0,05)$ en el tratamiento con mulch de vicia $(31,8 \mathrm{t} / \mathrm{ha})$ seguido por el mulch de avena (18,6 t/ha). El mulch de maíz (11,4 t/ha) obtuvo una producción similar al control sin mulch (13,9 t/ha), pero el tratamiento con mulch de caña de azúcar tuvo una menor producción ( 7,9 t/ha) que el control sin mulch, posiblemente debido a algún tipo de alelopatía. En cuanto al control de malezas, todos los tratamientos tuvieron un menor peso fresco de malezas $(\mathrm{p}<0,05)$ que el control sin mulch $(6,9 \mathrm{~kg})$, no obstante, el mulch de avena $(3,4 \mathrm{~kg})$ fue menos efectivo que el mulch de vicia $(2,4 \mathrm{~kg})$ y caña de azúcar $(1,9 \mathrm{~kg})$. El porcentaje de humedad y la concentración de nitrógeno fueron similares en todos los tratamientos. Los resultados ponen de manifiesto que la mejor opción para el cultivo de brócoli es el mulch de vicia ya que consiguió un aumento en la producción y un control eficiente de malezas.

Palabras clave: avena, caña de azúcar, maíz, vicia, control de malezas.

\section{ABSTRACT}

In a broccoli (Brassica oleracea $L$. var. Italica) culture of the ecuadorian Andes (2850 m.s.n.m., $01^{\circ} 24^{\prime}$ S, $78^{\circ} 35^{\prime}$ O), some variables of growth, crop production, weed control and moisture and nitrogen content, were evaluated in response to the application of different types of organic mulch. A randomized experimental block design with five treatments and three replicates was used. The treatments were: M0: without mulch; MM: maize mulch (Zea mays L.); MV: vicia mulch (Vicia sativa L.); MA: oat mulch (Avena sativa L.) and MC: sugarcane mulch (Saccharum officinarum L.). Vegetative growth and crop production were higher $(p<0.05)$ in vetch mulch treatment $(31.8 \mathrm{t} / \mathrm{ha})$ followed by oat mulch $(18.6 \mathrm{t} / \mathrm{ha})$. Corn mulch $(11.4 \mathrm{t} / \mathrm{ha})$ obtained similar crop production than control without mulch (13.9 t/ha), but sugar cane mulch treatment had lower production (7.9 t/ha) than control without mulch, probably due to some sort of allelopathy. As for weed control, all treatments had a lower fresh weight of weed $(p<0.05)$ than control without mulch $(6.9 \mathrm{~kg})$, however oat mulch $(3.4 \mathrm{~kg})$ was less effective than vetch mulch $(2.4 \mathrm{~kg})$ and sugar cane mulch $(1.9 \mathrm{~kg})$. Moisture and nitrogen concentration were similar in all treatments. The results show that the best option for growing broccoli is vetch mulch as it achieved an increase in production and an efficient weed control.

Key words: oat, sugar cane, corn, vetch, weed control.

\section{Introducción}

La agricultura convencional se basa en el uso de grandes cantidades de fertilizantes y pesticidas y aunque, en principio, permite un aumento de la producción, también es responsable de diversos problemas asociados como la disminución de materia orgánica, la erosión del suelo o la contaminación

\footnotetext{
1 Facultad de Ciencias Agropecuarias. Universidad Técnica de Ambato (UTA). Sector El Tambo-La Universidad, vía a QueroCevallos, Tungurahua, Ecuador.

2 Unidad Operativa de Investigación en Ciencias Agropecuarias. UTAmbato. Tungurahua, 1801334, Ecuador.

* Autor para correspondencia: da.risco@uta.edu.ec
}

Fecha de Recepción: 22 Agosto, 2016.

Fecha de Aceptación: 30 Octubre, 2016.

DOI: $10.4067 / \mathrm{S} 0718-34292016005000038$. 
ambiental (Rice et al., 2001). Por estos motivos, en las últimas décadas existe un interés creciente en el desarrollo de prácticas de cultivo alternativas que sean sustentables y requieran un menor uso de insumos externos (Allen et al., 1991).

El mulch o acolchado es una práctica que consiste en cubrir la superficie del suelo con diferentes materiales plásticos u orgánicos con el objetivo de prevenir pérdidas de humedad, reducir la presencia de malezas, mantener una temperatura del suelo constante y promover la productividad del suelo (Abdul-Baki y Teasdale, 1994). Los mulches plásticos son muy utilizados, aunque presentan algunas desventajas como un aumento en el volumen de escorrentía, un mayor costo asociado y la generación de residuos no degradables a corto plazo (Duppong et al., 2004). Por el contrario, los mulches orgánicos proporcionan beneficios adicionales al suelo como un aumento en la materia orgánica, una mejora en la retención de humedad y en las propiedades físicas y químicas del suelo (Teasdale y Mohler, 1993). Los mulches orgánicos a menudo consisten en materiales que en otros casos son considerados como residuos, pero cuando se usan como acolchado producen mejoras para el cultivo y el suelo. Los mulches de cereales y leguminosas son ampliamente utilizados en diferentes sistemas de cultivo. El uso de leguminosas se debe principalmente al enriquecimiento del nitrógeno del suelo fijado biológicamente, mientras que el mulch de cereales se utiliza por su capacidad para reducir la lixiviación de nitrógeno y suprimir malezas (Hooker et al., 2008).

El brócoli es uno de los cultivos no tradicionales más importantes de los Andes ecuatorianos, de tal forma que este país se ha convertido en los últimos años en el principal exportador de Sudamérica. Pertenece a los cultivos de col y su importancia radica en que posee un alto valor nutricional y comercial (Yoldas et al., 2008). Se han estudiado los efectos de diferentes tipos de mulch orgánico, aplicado directamente como mulch, o como residuos de cultivos de cobertura, en distintos cultivos de col. Así, por ejemplo, Bottenberg et al. (1997) obtuvieron una menor producción en repollo con mulch de avena en comparación con el laboreo convencional; Abdul-Baki et al. (1997a) encontraron que la producción de brócoli crecido en mulch de soya, mijo y una mezcla de ambas fue igual a la producción del control crecido convencionalmente; y Kosterna (2014) obtuvo un aumento en la producción de brócoli utilizando mulch de centeno, maíz, canola y trigo sarraceno.

Otro motivo por el que se utiliza el mulch en agricultura es su efecto sobre el control de malezas (Gupta, 1991). Mediante el bloqueo de la luz, la liberación de aleloquímicos y la disminución de la temperatura del suelo, el mulch orgánico puede suprimir la germinación y el crecimiento de malezas (Moore et al., 1994).

El objetivo del presente estudio fue estudiar el efecto de diferentes tipos de mulch orgánico (vicia, avena, maíz, caña de azúcar) sobre el crecimiento y la producción de brócoli, y sobre el control de malezas, en la provincia de Tungurahua, Ecuador.

\section{Materiales y Métodos}

\section{Localización y tratamientos}

La investigación se realizó entre enero y abril de 2015 en la Facultad de Ciencias Agropecuarias de la Universidad Técnica de Ambato, a $2.850 \mathrm{msnm}$. ( $01^{\circ}$ $\left.24^{\prime} \mathrm{S}, 78^{\circ} 35^{\prime} \mathrm{O}\right)$. El clima de la zona es ecuatorial de alta montaña, con una alta variación diaria de la temperatura y una baja variación estacional (Pourrut et al., 1995). Las condiciones climáticas durante el ensayo fueron medidas en una estación climática situada en la universidad (Tabla 1). El suelo de la zona de estudio es un Andisol (Soil Survey Staff, 2006) derivado de cenizas volcánicas. Las propiedades fisicoquímicas del suelo antes del ensayo fueron: textura franco arenosa, $\mathrm{pH} 6,49$, materia orgánica $4,50 \%$, nitrógeno total $64,2 \mathrm{ppm}$, fósforo asimilable $38,0 \mathrm{ppm}, \mathrm{K}$ asimilable $38,0 \mathrm{meq} / 100 \mathrm{~g}$, Ca asimilable 6,8 meq/100 g, Mg asimilable 2,4 meq/100.

Como material de siembra se utilizó brócoli (Brassica oleracea L. var. Italica) híbrido Avenger, con una densidad de plantación de $40 \mathrm{~cm}$ entre plantas y $70 \mathrm{~cm}$ entre hileras. Cada

Tabla 1. Condiciones climáticas durante el ciclo de cultivo en la Facultad de Ciencias Agropecuarias de la Universidad Técnica de Ambato, Ecuador.

\begin{tabular}{lccc}
\hline & $\begin{array}{c}\text { Temperatura media } \\
\left({ }^{\circ} \mathrm{C}\right)\end{array}$ & $\begin{array}{c}\text { Humedad relativa } \\
(\%)\end{array}$ & $\begin{array}{c}\text { Precipitación } \\
(\mathrm{mm})\end{array}$ \\
\hline Enero & 13,6 & 69,6 & 27,9 \\
Febrero & 14,8 & 63,8 & 27,2 \\
Marzo & 14,2 & 70,8 & 53,6 \\
Abril & 14,5 & 79,3 & 67,5 \\
\hline
\end{tabular}


parcela experimental consistió en 40 plantas, en un área de $11,2 \mathrm{~m}^{2}$, dispuestas en tres bloques cada uno con cinco tratamientos, con un área total de parcelas de $168 \mathrm{~m}^{2}$. La parcela útil, donde se tomaron todas las mediciones, consistió en 10 plantas en $4,48 \mathrm{~m}^{2}$.

Se manejó un diseño experimental de bloques al azar con cinco tratamientos y tres repeticiones. Los tratamientos fueron: M0: sin mulch; MM: mulch de maíz (Zea mays L.); MV: mulch de vicia (Vicia sativa L.); MA: mulch de avena (Avena L.) y MC: mulch de caña de azúcar (Saccharum officinarum L.). La aplicación del mulch se realizó una semana antes de la siembra del brócoli. El peso de todos los tratamientos fue de 12,5 t/ha con un espesor de $3-5 \mathrm{~cm}$. Los mulches de vicia, avena y caña de azúcar se extendieron directamente al suelo, donde comenzaron su proceso de descomposición. El mulch de maíz fue incorporado triturado, debido al alto contenido de humedad de la planta, para facilitar su descomposición. El abono, el control fitosanitario y el riego se realizaron en dosis iguales para todos los tratamientos conforme a las necesidades del cultivo.

\section{Mediciones}

En las plantas seleccionadas se midió: longitud del tallo a 30, 60 y 87 días después de siembra (DDS), número de hojas a 30, 60 y 87 DDS, peso y diámetro de la pella en cosecha y volumen radicular en cosecha.
En cosecha, se recolectaron dos pellas por parcela experimental, se pesaron, se secaron a $105^{\circ} \mathrm{C}$ en estufa durante 24 horas y se volvieron a pesar para el cálculo del contenido de humedad. En estas mismas muestras se analizó la concentración de nitrógeno total con un analizador elemental (LECO CHN628).

La presencia de malezas se estudió mediante un deshierbe a los 45 días, se pesó la materia fresca de malezas de cada una de las parcelas experimentales y se identificaron los principales tipos de malezas presentes en el cultivo.

\section{Análisis estadístico}

El análisis de datos se realizó mediante análisis de varianza utilizando el paquete estadístico INFOSTAT (www.infostat.com.ar). Cuando existieron diferencias entre tratamientos se aplicó el test de Tukey $(\mathrm{p}<0,05)$.

\section{Resultados y Discusión}

\section{Parámetros vegetativos}

Los distintos tipos de mulch influyeron de forma significativa $(\mathrm{p}<0,05)$ en el crecimiento vegetativo de brócoli (Figura 1). La longitud del tallo fue mayor en los tratamientos con mulch de vicia (MV) y avena (MA), y a partir de los 60 DDS, MV fue mayor que el resto de tratamientos. El tratamiento

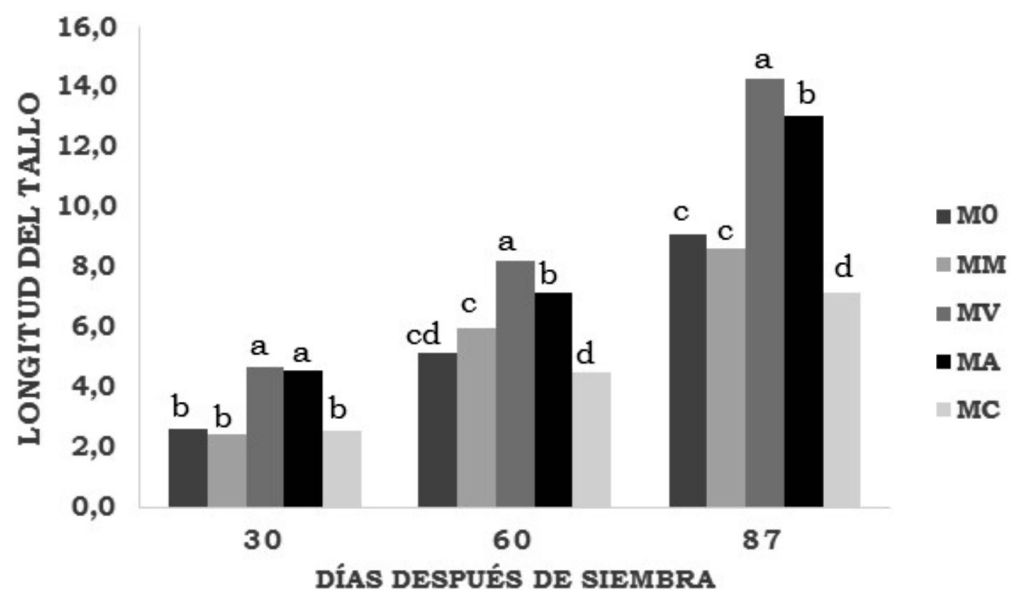

Figura 1. Longitud del tallo de plantas de brócoli por efecto de la aplicación de diferentes tipos de mulch orgánico. Diferencia mínima significativa de acuerdo con la prueba de Tukey. Barras con una letra común no son significativamente diferentes $(p<0,05)$. M0: Control sin mulch; MM: Mulch de maíz; MV: Mulch de vicia; MA: Mulch de avena; MC: Mulch de caña de azúcar. 
con mulch de caña de azúcar (MC) tuvo una menor longitud del tallo que el control sin mulch (M0) desde los 60 DDS (Figura 1). El número de hojas también fue superior para los tratamientos MV y MA aunque las diferencias comenzaron a partir de los 60 DDS y el tratamiento MC tuvo menor número de hojas que M0, en este caso a partir de la primera medición (Figura 2). Según Sinkeviciene et al., (2009) un aumento de la temperatura más lento bajo mulches en el periodo del crecimiento de las plantas contribuye a un mejor desarrollo vegetativo, aunque en el caso de los tratamientos MC y MM pudieron influir otros factores, como algún tipo de alelopatía, que incidieron en su menor crecimiento.

\section{Parámetros de cosecha}

Los parámetros de producción fueron afectados $(\mathrm{p}<0,05)$ por los diferentes tipos de mulch orgánico
(Tabla 2). El mayor peso y diámetro de pella, así como el volumen radicular, ocurrió en el tratamiento MV seguido de MA. El tratamiento MM obtuvo resultados similares al tratamiento sin mulch (M0) en peso de pella y volumen radicular, pero el tratamiento MC tuvo un menor peso de pella y volumen radicular que el tratamiento M0. La interacción entre el tipo de mulch y el cultivo es de suma importancia para la producción final del cultivo. Schonbeck et al. (1993), obtuvieron una producción de repollo reducida por el sistema de mulch con centeno, mientras que Kosterna (2014) consiguió una mayor producción de pellas de brócoli utilizando mulch de maíz, lo que contradice nuestros resultados.

Otro efecto que produjeron los mulch de vicia $\mathrm{y}$ avena fue un adelanto en la maduración del cultivo. Se realizaron dos cosechas a los 87 y 93 DDS; primero se cosecharon las parcelas con mulch de vicia y avena y posteriormente se cosecharon

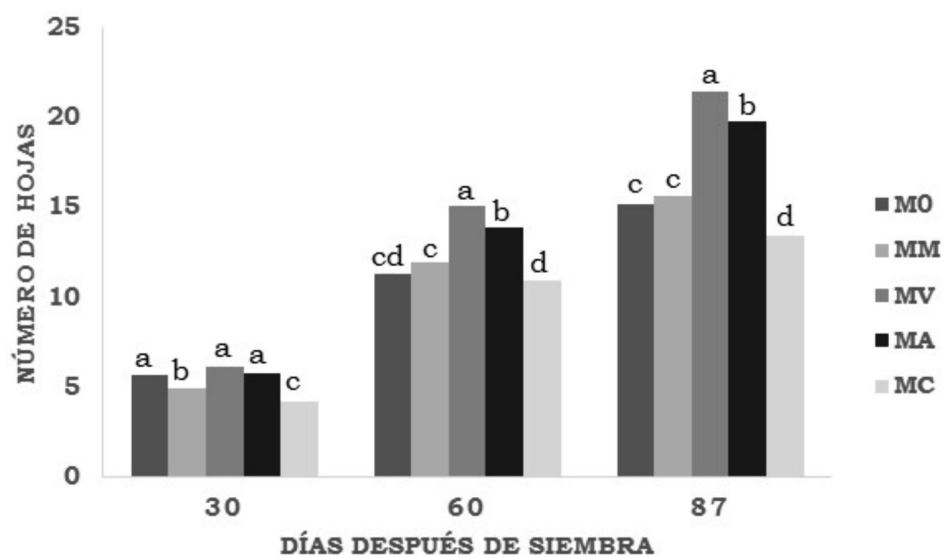

Figura 2. Número de hojas de plantas de brócoli por efecto de la aplicación de diferentes tipos de mulch orgánico. Diferencia mínima significativa de acuerdo con la prueba de Tukey. Barras con una letra común no son significativamente diferentes (p < 0,05). M0: Control sin mulch; MM: Mulch de maíz; MV: Mulch de vicia; MA: Mulch de avena; MC: Mulch de caña de azúcar.

Tabla 2. Parámetros de cosecha en brócoli por efecto de diferentes tipos de mulch orgánico. Diferencia mínima significativa de acuerdo con la prueba de Tukey. Columnas con letra común no son significativamente diferentes $(\mathrm{p}<0,05)$. M0: Control sin mulch; MM: Mulch de maíz; MV: Mulch de vicia; MA: Mulch de avena; MC: Mulch de caña de azúcar.

\begin{tabular}{lcccc}
\hline Tratamiento & $\begin{array}{c}\text { Peso pella } \\
(\mathrm{kg})\end{array}$ & $\begin{array}{c}\text { Diámetro pella } \\
(\mathrm{cm})\end{array}$ & $\begin{array}{c}\text { Producción } \\
(\mathrm{t} / \mathrm{ha})\end{array}$ & $\begin{array}{c}\text { Volumen radicular } \\
\left(\mathrm{cm}^{3}\right)\end{array}$ \\
\hline M0 & $0,39 b c$ & $11,1 c$ & $13,9 b c$ & $141,3 c$ \\
MM & $0,32 c d$ & $11,1 c$ & $11,4 c d$ & $150,2 c$ \\
MV & $0,89 a$ & $22,2 a$ & $31,8 a$ & $227,0 a$ \\
MA & $0,52 b$ & $16,6 b$ & $18,6 b$ & $170,3 b$ \\
MC & $0,22 d$ & $10,6 c$ & $7,9 d$ & $126,7 d$ \\
E.E. & 0,04 & 0,51 & 0,04 & 2,24 \\
p-valor & $<0,0001$ & $<0,0001$ & $<0,0001$ & $<0,0001$ \\
\hline
\end{tabular}


las parcelas con mulch de maíz, caña de azúcar y control sin mulch. La precocidad de un cultivo es a menudo extremadamente importante en la venta y rentabilidad de muchos cultivos vegetales.

\section{Control de malezas}

Todos los tratamientos con mulch descendieron significativamente $(\mathrm{p}<0.05)$ el peso fresco de malezas en comparación al control sin mulch (Figura 3). Los tratamientos que más descendieron fueron MV y MC, en cantidades significativamente superiores a MA. Por el contrario, Campiglia et al. (2010) encontraron una mayor capacidad supresiva de malezas en la avena que en la vicia en un cultivo de tomate. El efecto supresivo del mulch sobre las malezas puede deberse tanto a efectos físicos como químicos. Los efectos físicos incluyen la inhibición de la germinación de las semillas mediante la alteración de su ambiente (cambios en la disponibilidad de luz, temperatura y humedad del suelo) (Teasdale y Mohler, 1993), mientras que los efectos químicos se deben a la liberación de aleloquímicos causados por la decomposición del mulch (Moonen y Barberi, 2006). En el caso del mulch de caña el efecto alelopático no se limitó solo a las malezas, sino que también produjo un descenso significativo en la producción del cultivo de brócoli (Tabla 2).

Entre las especies de malezas aparecidas en el cultivo con mulch se encontraron: Galinsoga parviflora, Chenopodium album, Malva silvestris, Plantago lanceolata, Pennisetum clandestinum y Taraxacum officinale.

\section{Contenido de humedad y nitrógeno}

No se observaron diferencias significativas en el contenido de humedad y nitrógeno entre los diferentes tipos de mulch (Tabla 3), aunque los tratamientos MV, MA y MC tuvieron un contenido de nitrógeno un $10-12 \%$ superior a los tratamientos M0 y MM. Los residuos de leguminosas aportan cantidades variables de nitrógeno orgánico al suelo (Quemada y Cabrera, 1995), mientras que los residuos de gramíneas reducen las pérdidas de nitrógeno en el perfil del suelo por lixiviación (Jackson, 2000).

Tabla 3. Contenido de humedad y nitrógeno en pellas de brócoli por efecto de diferentes tipos de mulch orgánico.

Diferencia mínima significativa de acuerdo con la prueba de Tukey. Columnas con letra común no son significativamente diferentes $(\mathrm{p}<0.05)$.

\begin{tabular}{lcc}
\hline Tratamiento & Humedad (\%) & Nitrógeno (\%) \\
\hline M0 & $87,2 a$ & $4,0 a$ \\
MM & $87,1 a$ & $4,0 a$ \\
MV & $87,9 a$ & $4,4 a$ \\
MA & $87,1 a$ & $4,4 a$ \\
MC & $86,9 a$ & $4,5 a$ \\
E.E. & 0,25 & 0,17 \\
p-valor & 0,11 & 0,16 \\
\hline
\end{tabular}

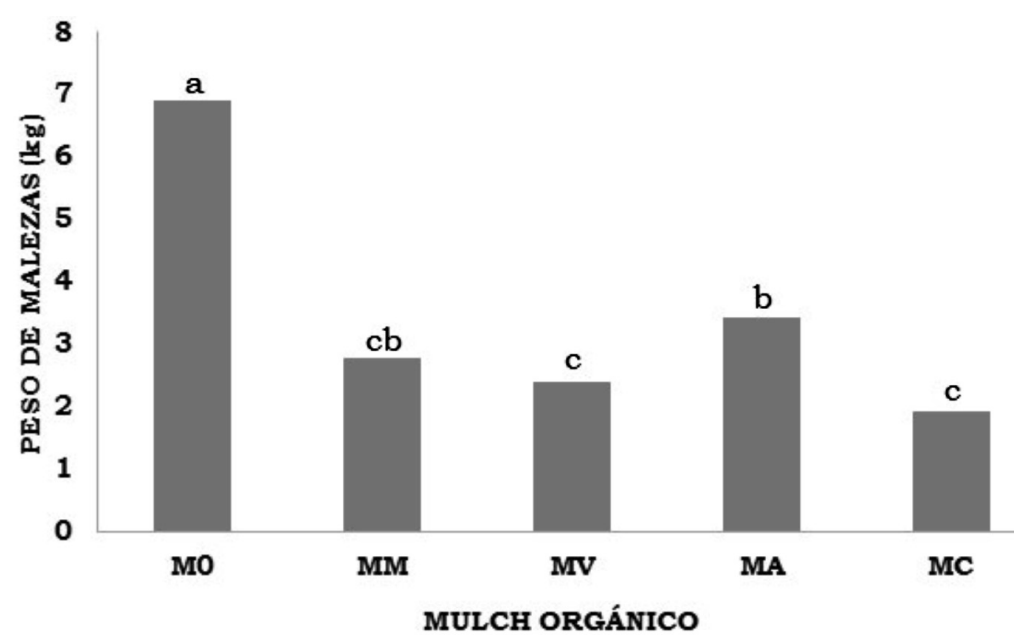

Figura 3. Peso de malezas (kg) en parcelas cultivadas de brócoli por efecto de la aplicación de diferentes tipos de mulch orgánico. Diferencia mínima significativa de acuerdo con la prueba de Tukey. Barras con una letra común no son significativamente diferentes ( $\mathrm{p}$ 0.05). M0: Control sin mulch; MM: Mulch de maíz; MV: Mulch de vicia; MA: Mulch de avena; MC: Mulch de caña de azúcar. 


\section{Conclusiones}

El uso de mulches orgánicos es una práctica que puede resultar eficiente para el aumento de la producción y el control de malezas en brócoli, dependiendo principalmente del tipo de mulch utilizado. En este estudio, el mulch de vicia consiguió un aumento en la producción de brócoli y un control más efectivo de las malezas, mientras que el mulch de avena también produjo un aumento en la producción pero un menor control de malezas que el mulch de vicia.
Por el contrario, el mulch de caña de azúcar produjo un control de malezas eficiente pero la producción se redujo a niveles menores que el control sin mulch.

\section{Agradecimientos}

Esta investigación fue realizada bajo el proyecto 1246 - CU - P - 2014 de la Universidad Técnica de Ambato (Ecuador). Los autores quieren agradecer a los operarios agrícolas del Campus Querochaca por su ayuda en los trabajos de campo.

\section{Literatura Citada}

Abdul-Baki, A.; Teasdale, J.R.

1994. Sustainable production of fresh-market tomatoes with organic mulches. USDA/ARS Bulletin FB-2279, 9 p.

Abdul-Baki, A.; Morse, R.D.; Devine, T.E.; Teasdale, J.R.

1997. Broccoli production in forage soybean and foxtail millet cover crop mulches. Horticulture Science 32 (5): 836-839.

Allen, P.A.; Van Dusen, D.; Lundy, J.L.; Gliessman, S.R.

1991. Integrating social, environmental and economic issues in sustainability. American Journal of Alternative Agriculture 6: 34-39.

Bottenberg, H.; Masiunas, J.; Eastman C.; Eastburn, D.

1997. Yield and quality constraints of cabbage planted in rye mulch. Biological Agriculture \& Horticulture 14 (4): 323-342.

Campiglia, E.; Mancinelli, R.; Radicetti, E.; Caporali, F. 2010. Effect of cover crops and mulches on weed control and nitrogen fertilization in tomato (Lycopersicon esculentum Mill.). Crop Protection 29: 354-363.

Duppong, L.M.; Delate, K.; Liebman, M.; Horton, R.; Romero,

F.; Kraus, G.; Petrich, J.; Chowdbury, P.K.

2004. The effect of natural mulches on crop performance, weed suppression and biochemical constituents of Catnip and St. John's Wort. Crop Science 44 (3): 861-869.

Gupta, G.N.

1991. Effects of mulching and fertilizer application on initial development of some tree species. Forest Ecology and Management 44: 211-221.

Hooker, K.V.; Coxon, C.E.; Hackett, R.; Kirwan, L.E.; O’Keeffe,

E.; Richards, K.G.

2008. Evaluation of cover crop and reduced cultivation for reducing nitrate leaching in Ireland. Journal of Environmental Quality 37: 138-145.

Jackson, L.E.

2000. Fates and losses of nitrogen from a nitrogen-15-labeled cover crop in an intensively managed vegetable system. Soil Science Society of America Journal 64: 1404-1412.

Kosterna, E.

2014. The yield and quality of broccoli grown under flat covers with soil mulching. Plant Soil Environment. 60 (5): 228-233.

Moonen, A.C.; Barberi, P.

2006. An ecological approach to study the physical and chemical effects of rye cover crop residues on Amaranthus retroflexus, Echinocloa crus-galli and maize. Annals of Applied Biology 148: 73-89.

Moore, M.J.; Gillespie, T.J.; Swanton, C.J.

1994. Effect of cover crop mulch on weed emergence, weed biomass, and soybean (Glycine max) development. Weed Technology 8: 512-518.

Pourrut, P.; Gómez, G.; Bermeo, A.; Segovia, A.

1995. Factores condicionantes de los regímenes climáticos e hidrológicos. En: Pourrut, P. Estudios de Geografía. El agua en el Ecuador. Clima, precipitaciones, escorrentía. Colegio de Geógrafos del Ecuador. Corporación Editora Nacional, Quito, Ecuador, 7-12 p.

Quemada, M.; Cabrera, M.L.

1995. Carbon and nitrogen mineralized from leaves and stems of four cover crops. Soil Science Society of America Journal 59: 471-477.

Rice, P.J.; McConnell, L.L.; Heighton, L.P.; Sadegui, A.M.; Isensee, A.R.; Teasdale, J.R.; Abdul-Baki, A.A.; Harman-Fetcho, J.A.; Hapeman, C.

2001. Runoff loss of pesticides and soil: a comparison between vegetative mulch and plastic mulch in vegetable production systems. Journal of Environmental Quality 8: 767-770

Schonbeck, M.; Herbert, S.; DeGregorio, R.; Mangan, F.;

Guillard, K.; Sideman, E.; Herbst, J.; Jaye, R.

1993. Cover cropping system in the Northeastern United States: 1. Cover crop and vegetable yields, nutrients and soil conditions. Journal of Sustainable Agriculture 1: 21-35.

Sinkeviciene, A.; Jodaugiene, D.; Pupaliene, R.; Urboniene, M. 2009. The influence of organic mulches on soil properties and crop yield. Agronomy Research 7 (I): 485-491.

Soil Survey Staff (SSS)

2006. Keys to soil taxonomy. Tenth edition. USDA. Washington D.C., $332 \mathrm{p}$.

Teasdale, J.R.; Mohler, C.L.

1993. Light transmittance, soil temperature and soil moisture under residue of hairy vetch and rye. Agronomy Journal 85: 673-680.

Yoldas, F.S.; Ceylan, B.; Yagmur Y.; Mordogan, N.

2008. Effect of nitrogen fertilizer on yield quality and nutrient content in broccol. Journal of Plant Nutrition 31: 13-43. 